\title{
Wi-Fi Calling
}

Dhaval Gandhi

U.G Student
EXTC
DJSCOE,
Mumbai- India

\author{
Jay Karmarkar \\ U.G Student \\ EXTC \\ DJSCOE \\ Mumbai- India
}

\author{
Aarushi \\ Jalundhwala \\ U.G Student \\ EXTC \\ DJSCOE, \\ Mumbai- India
}

\author{
Shivani \\ Bhattacharjee \\ Assistant Professor \\ EXTC \\ DJSCOE, \\ Mumbai- India
}

\begin{abstract}
In this paper, we aim to study the Generic Access Network System or GAN. Generic Access Network is a telecommunications system that extends mobile voice, data and multimedia (IMS/SIP) applications over IP networks. Unlicensed Mobile Access or UMA is the commercial name used by mobile carriers for external IP access into their core networks. More recently, the system has been called Wi-Fi Calling. Wi-Fi Calling enables to make and receive calls, send messages and check voicemails even when there is no mobile reception. The cause for the increasing popularity of Wi-Fi Calling is that it extends your coverage and it has no additional charge.
\end{abstract}

Keywords: Generic Access Network, LTE, VANC, VoLGA, 3GPP.

\section{INTRODUCTION}

Wi-Fi Calling is based on a Smart Wi-Fi Application from Kineto Wireless. It uses Wi-Fi to provide better mobile phone coverage.Several operators around the world have Wi-Fi Calling services, including T-Mobile US, Orange UK and Rogers Wireless in Canada.

The concept of the Wifi Calling application is based on Generic Access Network. Instead of using a conventional 2G or $3 \mathrm{G}$ network, a data network is used to establish a call. The conventional network (i.e. packet switched access network) has some shortcomings due to which calls may not be successfully conducted in some cases. To overcome this and to provide network coverage anywhere, anytime the concept of Voice over LTE was brought forward. Explained below is the conventional network and its shortcomings as well as the proposed solution, VoLGA.

\section{LTE}

1. The next generation network technology LTEor Long Term Evolution was used in order to keep up with the demand of rising use of mobile telecommunications network.

2. It is a project of Third Generation Partnership Project (3GPP) and offers an upgrade path for all major third generation technologies.

3. The major disadvantage of this packet switched wireless network is that voice call and SMS are not available in LTE as there are based on circuit switched radio and core network infrastructure.

4. One solution designed was to allow fallback to $2 \mathrm{G}$ for circuit switched services such as voice calls.

5. The problem with this is longer call setup times which result in degradation of user experience.
6. The other solution that was proposed was Voice over LTE via Generic Access Network (VoLGA). The concept is to the concept is to connect the already existing Mobile Switching Centers to the LTE network via a gateway.

7. As no fallback to a legacy network is required, call setup times are not increased and the user's quality of experience is consistent with that of the $2 \mathrm{G}$ or $3 \mathrm{G}$ voice environment

\section{VoLGA}

1. VoLGA is based on the existing 3GPP Generic Access Network (GAN) standard. The purpose of GAN is to extend mobile services over a generic IP access network.

2. One of the popular applications of GAN is with WiFi-enabled phones.

3. On the network side, VoLGA only requires software enhancements to the circuit to packet gateways which already exist for GAN. No modifications are required on the MobileSwitching Centres or the LTE core and access network nodes.

4. On the mobile device side, the protocol stack initially developed for GAN can also be re-used in large parts.

5. VoLGA requirements: GAN requires dual mode mobile devices which have both a GSM/UMTS radio interface and a $\mathrm{Wi}-\mathrm{Fi}$ radio interface. Such mobile devices are available today from almost all mobile manufacturers.

6. A GAN gateway securely connects a subscriber to the infrastructure of a network operator and voice calls and other circuit switched services such as SMS are then securely transported between the mobile device and the Gateway over the intermediate Wi-Fi link and Internet access network.

7. From a mobile devicepoint of view there is not much difference between the two access methods because both networks are based on IP.

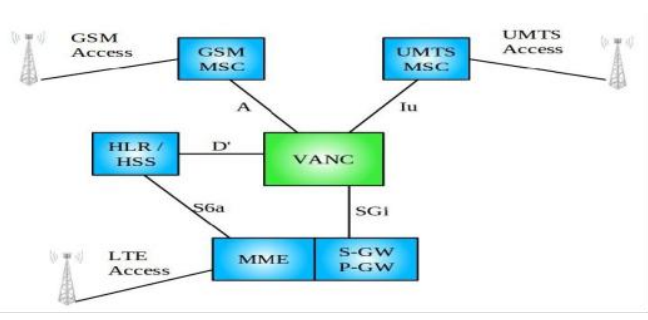

Fig 1: Basic VoLGA network setup 


\subsection{VoLGA in the Network}

Figure 1 is an overview of the basic network setup for VoLGA in the home network as described in the Voice over LTE via Generic Access Stage 2 specification7. For an easy start, there are interfaces to improve quality of service in the LTE network and to handle on-going voice calls to a circuit switched network..

The only new network element introduced is the VoLGA Access Network Controller(VANC). All other network elements and the interfaces between them already exist and are used without any modifications.

\subsection{VoLGA from the LTE Network Point of View}

On the LTE side, the VANC connects to the Packet Data Network Gateway (P-GW) via the standard SGi interface. Both signalling and user data traffic (i.e. the voice packets) are transported over this interface.

\subsection{VoLGA from the Circuit Switched Network Point of View}

On the circuit switched network side the A-interface is used to connect the VANC to a GSM Mobile Switching Centre (MSC). The Iu-interface is used to connect the VANC to the UMTS MSC. The VANC thus looks like a GSM Base Station Controller (BSC) to a GSM MSC and like a UMTS Radio Network Controller (RNC) to a UMTSMobileSwitchingCentre. Which interface is used in practice depends on the requirements of the network operator. As the $\mathrm{A}$ and Iu interfaces are used without any enhancements, the MSCs are not aware that the mobiles are not directly connected via their respective radio networks but instead are connected over LTE. Consequently, no changes are required on these network nodes to support voice, SMS and other services over the LTE network.

\section{REGISTERING TO THE NETWORK}

When a mobile device is switched on and detects an LTE network it first registers with the Mobility Management Entity (MME) over the LTE access network. The MME uses the Home Location Register / Home Subscriber Server (HLR/HSS) to retrieve the subscriber data required for authenticating and managing the user.

After registering with the LTE network, the mobile then establishes a connection to theVANC. First, a suitable IP connection needs to be in place. In the home network the default bearer might be used. It is also possible to use a separate bearer and IP address for the purpose. Once the IP address of the VANC is known, the mobile establishes a secure IPSec tunnel to it over the LTE network. During the process the VANC authenticates the user with the help of authentication information stored inthe HLR/HSS.

Next, the mobile device registers to the MSC through the secure tunnel and the VANC. The Direct Transfer Application Part (DTAP) protocol is used for this purpose, which is already known from GSM and UMTS. Merely the VANC adds information such as a cell-id (2G) or the service area identifier $(3 \mathrm{G})$ to the initial registration message as defined in the GSM and UMTS standards respectively.

\section{OUTGOING CALLS OVER LTE}

The VANC is linked to the user using a Wi-Fi signal and to the MSC of a UMTS network using either the A or Iu interface. For an outgoing call using LTE, the MSC thinks of the VANC as a BSS, as it would in a UMTS network. First, the mobile device sends a request to the VANC to activate the connection and form a dedicated link. The VANC then contacts the MSC to form a dedicated connection in order to process the call. The MSC after receiving this message authenticates the user using ciphering techniques. The mobile device sends a message after the link to the MSC has been set up. This message contains, among other things, the phone number that is to be called. The MSC acknowledges this request and sends an Assignment Request message to the VANC like it would to the BSS in the UMTS network. The VANC translates this message and activates the channel through which IP packets containing voice data will be transmitted. The VANC then signals the MSC to show completion in establishment of a 'pseudo' channel. The MSC sends Alert and Connect messages to the mobile device to show readiness for voice call. The voice conversation can begin after the user device acknowledges these messages.

The voice signal is transmitted in different ways depending upon the network MSC used to secure a connection. For a GSM MSC, the message is transmitted in a 64kbps TDM timeslot. For a UMTS MSC, the signal is transmitted via an ATM or IP based data flow. The VANC is responsible for converting the voice data stream into IP packets. The Real Time Transfer (RTP) protocol is used for this purpose.

\section{INCOMING CALLS OVER LTE}

For an incoming call received via LTE, the MSC first has to establish a connection to the users mobile device. The MSC sends a paging request to the VANC. The VANC itself is already connected to the mobile device of the receiver, and thus forwards the paging message straight to the user device. The VANC sends this page via the established IPSec channel which is transparent to the LTE network. The mobile in question may have one of two active states. If the status of the device for the LTE network is 'active', the packet is delivered immediately. If the device status is inactive for the LTE network, the location where the device was last in contact with the network is used as a reference. The area over which the network scans to locate the user is called as the tracking area. A LTE paging message is sent via all the base stations in the vicinity of the area where the device was last located. After the paging message is received by the device, it gets re connected to the LTE network and the data packet through the IPSec channel is delivered to it. The time required for the LTE paging is similar to the time required to page the mobile device in a circuit switched wireless network. Therefore, thecall establishment time for a voice call over LTE is similar to that of a GSM or UMTS network.

\section{HANDOVER}

A handover is the method by which the call of a user does not fluctuate or get disconnected when he moves out of range for a particular cell. In modern day systems, the mobile device is constantly reading the signal strength levels from all the base stations in its vicinity. If the power from the currently active base station falls below a particular level, the procedure for handover is initiated. The base station with the highest signal strength is selected and the mobile device gets connected to that base station and the call continues without any problem to the user. This is called a handover.

A very crucial feature of VoLGA is its ability to conduct a handoff like in a conventional UMTS network when the mobile device leaves the range of a LTE network. This is one of the most valuable differentiators that network operator supplied voice services have compared to over-the-top VoIP services 
The steps for a handover are as follows:

1. When the mobile registers with the LTE network it signals its capability to the MME. The network is thus aware that this procedure needs to be executed when the mobile device is about to leave the LTE coverage area while a bearer is active.

2. When a base station finds that the device could be better served by a $2 \mathrm{G}$ or $3 \mathrm{G}$ network rather than a LTE, it instructs the surrounding cells to check the signal strength independently.

3. The MME informs the VANC about the impending handoff that is required. The message it sends includes information like the id of the target cell and the identity of the subscriber for whom the handover is to be executed.

4. The VANC then uses this message and readies a standard GSM or UMTS handoff message to be sent to the target cell. If the target cell is connected to the same MSC as the VANC, the cell is prepared for the handoff locally. For the MSC, this type of handoff is no different from a regular handoff. If the target cell belongs to a different MSC, standard Inter MSC handoff procedure is followed.

\section{QUALITY OF SERVICE}

Another important feature that sets VoLGA apart is its ability to implement Quality of Service measures to ensure that the bandwidth required for the call remains reserved and dedicated to a user.

To activate QoS for a call the VANC contacts the PCRF and requests that packets to and from certain IP addresses and UDP ports be given a higher priority in the network. Based on the subscription profile of the user in the HLR/HSS, the request is granted or denied. If granted, the PCRF establishes a secondary bearer throughout the LTE network and also informs the mobile device. Packets matching the criteria set above are then given a preferential treatment by all network components and also the mobile device as uplink capacity might also be limited.

\section{INTERNATIONAL ROAMING}

\subsection{Local Breakout}

When VoLGA is natively supported in the roaming network, the mobile uses a VANC and MSC in the visited network. Interfaces that already exist today for standard international GSM and UMTS roaming are used for obtaining subscriber's subscription. For billing, the standard international billing procedure is used. A roaming subscriber always contacts a $2 \mathrm{G}$ or 3G Gateway GPRS support node (GGSN) in the home network. This helps the user keep his device configuration while roaming. But all the IP information is routed to the home network which causes a few problems.

For a visiting network, a feature called local breakout is used. It is used sparingly. Local breakout allows the use of a GGSN (in case of GSM and UMTS) in the visited network and is controlled by using a visited network specific Access Point Name (APN). A default APN is specified which is used if the foreign network is not in the list.

\subsection{Home Network Routing}

In case the visited network does not support VoLGA natively it is possible to use an APNthat establishes a connection to the home network of the subscriber. This is what happens today for a standard IP connection for mostusers while in roaming status. However there areseveral disadvantages to this scheme:

1. The voice packets need to be routed back to the home network. This causes an unnecessary increase in the amount of data to be transmitted.

2. International roaming is disabled.

3. Emergency calls are not possible in this setup, as a home network MSC is used thatcan't connect the call to an emergency center in the visited country.

Despite these disadvantages it might still be favourable to provide VoLGA services from the home network in visited networks that do not support it natively.

\section{PRESENT AND FUTURE ALTERNATIVES}

VoLGA is not the only system designed to deliver voice and SMS services over LTE networks.

\subsection{CS Fallback (CSFB)}

The approach favoured by many 3GPP members as an initial solution for delivering voice and SMS services over LTE is 'circuit switched fallback', which is specified in TS 23.27210. The idea behind this solution is to use a $2 \mathrm{G}$ or $3 \mathrm{G}$ network for incoming and outgoing calls, i.e. the mobile has to leave the LTE network for making or accepting voice calls. Disadvantages of CSFB are as follows: Changing to another network takes time, which has an adverse effect on call setup times. Already today, users perceive mobile call setup times as too long. It is estimated that even in the best case scenario, both mobile originating and mobile terminating call establishment times would increase by at least 1.5 seconds. From a network point of view a new MSC and SGSN interface is required to signal incoming calls and SMS messages to the MME. This interface is referred to as SGs in the standards and is based on IP and therefore requires new software on network nodes that are delivering the main services today. Also, many network operators have bought MSCs and SGSNs from different vendors, further increasing cost and interoperability testing. On the positive side, no fallback to a $2 \mathrm{G}$ or $3 \mathrm{G}$ network is required for delivering SMS messages.

\subsection{IMS}

The IP Multimedia Subsystem or IMS has its core based on the popular Session Initiation Protocol (SIP) which is widely used in fixed line IP based networks for Voice over IP. For wireless networks, many additions were specified like for example features to handle wireless specific issues such as unreliable radio connections, application servers for external application development, international roaming, scalability, security, etc. Given the current state, it is unlikely that an IMS solution is available in the first years of LTE to serve as a voice platform. Therefore, IMS will not be an immediate alternative to VoLGA. The initial concept of IMS was to be an IP platform only, i.e. no ties were specified for services to roam between an IP network and a circuit switched legacy network. Over time, it was recognized that this approach is not suitable for a practical deployment and extensions have been standardized to enable ongoing voice sessions to be handed over between an IP based wireless access network and a circuit switched access network. The latest of these features is Single Radio Voice Call Continuity (SRVCC). As the somewhat long name of the feature implies that only a single radio in the mobile device has to be active on at any one time, 
even during a handover. This simplifies mobile device development. 3GPP had the foresight to specify SRVCC in an IMS independent manner. Therefore, the VoLGA forum decided to use it as the means to handover VoLGA calls from LTE to GSM or UMTS. As a result, no VoLGA specific features are required in the MSC or SGSN for VoLGA, which is a great plus for deployment in a running network.

\subsection{Over-the-Top VoIP}

Some network operators might also decide to go an entirely different way and offer voice services over LTE with external partners. UK network operator '3', for example, has partnered with Skype15 to deliver voice services in addition to their own circuit switched services. While their Skype client also uses circuit switched resources for the time being this could change quickly in the future. There are two technical disadvantages of partnering with external voice service providers, however. The first one is that external voice service providers have no control over quality of service in the wireless network and thus they can't ensure a good quality of experience under all load situations. A potential solution to this issue could be to install logic in the network to ensure quality of service for data streams that are recognized to belong to an external voice service the user has subscribed to. PCRF functionality could be used for this as described earlier but there is no standardized way to offer this yet. The second problem with Over-the-Top VoIP is that calls can't be handed over to a circuit switched $2 \mathrm{G}$ or $3 \mathrm{G}$ network when a user leaves the LTE coverage area. This occurs because external applications can't be tied into the wireless network infrastructure easily. This is a serious disadvantage, as LTE networks will have an inferior network coverage compared to GSM.

\section{ADVANTAGES AND LIMITATIONS 11.1 Advantages}

This paper has shown that VoLGA has a number of significant advantages over other Voice over IP solutions for LTE. VoLGA does not require updating any of the existing network components, thus ensuring a very quick and smooth market introduction. Instead, all development is concentrated in the VoLGA Access Network Controller (VANC).

In addition, VoLGA enables other circuit switched services from day one without any additional development. One of those is SMS for person to person messaging, a significant revenue generator for mobile network operators. SMS is also used for updating the configuration of mobile devices and for transmitting mandatory roaming information messages. On the mobile devices side it is likely that VoLGA can be developed very quickly, as the already existing GAN protocol stack can be mostly re-used. The only major change in the software is handover handling, as the network based Single Radio Voice Call Continuity (SRVCC) feature will be used. VoLGA can also ensure a smooth introduction of global roaming. In case it is supported in the visited network, local breakout allows using the VANC and MSCs in the visited network. For network operators launching LTE as a data only network or only with voice options not supported by a mobile device, it is also possible to use the VANC and the MSCs of the home network.

\subsection{Limitations}

First, it is not fully standardized. This is expected shortly, however. As it is likely that the stage 3 specification will be based on the equivalent GAN stage 3 specification, vendors can already develop products without waiting for a final stage 3 specification being published by the VoLGA forum.

Second, VoLGA is currently not a work item in 3GPP. There are probably several reasons for this, one being that the number of features for Release 9 was already high and most members wanted to ensure the tight completion deadline was met which would have been more difficult with an extended project scope.

\section{CONCLUSION}

From a complexity point of view, VoLGA makes it very simple to leverage existing $2 \mathrm{G}$ and $3 \mathrm{G}$ circuit switched equipment in live networks for LTE. This is especially because no software enhancements are required on existing network nodes. Due to this and other advantages of VoLGA described above, we believes that VoLGA has the chance to become a widespread Voice over LTE solution and will ensure that two of the main revenue generators for network operators, voice calls and SMS will be available in LTE networks very early on. In the future, VoLTE may be used as an alternative to GSM or CDMA communication. If a unique ID can be presented to each device, the call via VoLTE can be performed in exactly the same fashion as a GSM/CDMA call. The ubiquitous data networks present everywhere could thus provide both data as well as voice services. Introducing VoLTE will reduce network complexity by preparing for the shutdown of the CS domain. Since LTE is much more spectral-efficient than $2 \mathrm{G} / 3 \mathrm{G}$ networks, the cost for each voice call will decline LTE offers the next stage in the evolution of the networks on which these users depend for mobile connectivity. Such a large addressable market ensures: maximum equipment vendor focus; a wide range of devices; economies of scale for operators and end users buying equipment; and support for international roaming.

\section{ACKNOWLEDGEMENT}

We would like to thank the respected principal Dr.HariVasudevan of D.J. Sanghvi College of Engineering for giving us facilities and providing a propitious environment for working in the college. We would also like to thank S.V.K.M for encouraging us in such co-curricular activities.

\section{REFERENCES}

[1] Voice over LTE via Generic Access; Stage 2 Specification; Phase 1 - Available at the VoLGA Forum website

[2] M. Sauter, -VoLGA - A Whitepaper\| 2009.

[3] Kineto Wireless

[4] VoLGA: Voice Over LTE via Generic Access, Kineto Wireless - A Whitepaper; March 2009.

[5] Oleg Stepaniuk, -Voice over LTE via Generic Access (VoLGA) as a possible solution of mobile network transformation - IEEE.

[6] An Introduction to LTE: LTE, LTE-Advanced, SAE and 4G Mobile Communications - Christopher Cox

[7] 3GPP Generic Access Network: 3GPP TS43.318, -Mobile Broadcasting with Wimax; Amitabh Kumar, 2009.

[8] Evolution from $3^{\text {rd }}$ generation Generic Access Networks to Voice over LTE via Generic Access.

[9] Mobile Communications - Jochen Schiller

[10] SavoGlisic - Advanced Wireless Networks: 4G Technologies.

[11] MiikkaPoiksela, HarriHolma - Voice Over LTE.

[12] StefaniaSesia, IssamToufik - LTE - The UMTS Long Term Evolution: From Theory to Practice. 